\title{
Spin chains for robust state transfer: Modified boundary couplings vs. completely engineered chains
}

\author{
Analia Zwick, ${ }^{1,2}$ Gonzalo A. Álvarez, ${ }^{1}$ Joachim Stolze, ${ }^{1}$ and Omar Osenda ${ }^{2}$ \\ ${ }^{1}$ Fakultät Physik, Technische Universität Dortmund, D-44221 Dortmund, Germany. \\ ${ }^{2}$ Facultad de Matemática, Astronomía y Física and Instituto de Física Enrique Gaviola, \\ Universidad Nacional de Córdoba, 5000 Córdoba, Argentina.
}

\begin{abstract}
Quantum state transfer in the presence of static disorder and noise is one of the main challenges in building quantum computers. We compare the quantum state transfer properties for two classes of qubit chains under the influence of static disorder. In fully engineered chains all nearest-neighbor couplings are tuned in such a way that a single-qubit state can be transferred perfectly between the ends of the chain, while in chains with modified boundaries only the two couplings between the transmitting and receiving qubits and the remainder of the chain can be optimized. We study how the disorder in the couplings affects the state transfer fidelity depending on the disorder model and strength as well as the chain type and length. We show that the desired level of fidelity and transfer time are important factors in designing a chain. In particular we demonstrate that transfer efficiency comparable or better than that of the most robust engineered systems can also be reached in chains with modified boundaries without the demanding engineering of a large number of couplings.
\end{abstract}

PACS numbers: 03.67.Hk, 03.65.Yz, 75.10.Pq, 75.40.Gb

\section{INTRODUCTION}

One of the main challenges on the road to practical quantum computers is the reliable transfer of quantum information between quantum gates [1]. The main source of problems is the vulnerability of quantum systems to perturbations due either to manufacturing imperfections or to interactions with the environment. Overcoming (or avoiding) these problems has motivated an intensive search for systems able to transfer information with high quality while at the same time requiring minimal control in order to avoid the introduction of errors [2, 3 ]. The problem of state transfer has received a lot of attention in the last decade in the context of quantuminformation processing; nevertheless, an early antecedent can be found in the work of Shore and co-workers, see, for example, the paper of Cook and Shore [4].

Spin chains are a promising class of systems to serve as reliable quantum communication channels [2, 3, 511]. Perfect state transfer (PST) without any dynamical control can be achieved by an infinity of engineered spinspin coupling configurations [11 18] for a spin chain of given length. Regrettably this amazing transfer fidelity comes at a high price in terms of the accuracy required to design each interaction to avoid the loss of information [3, 11, 19, 20].

In order to assess the reliability of these systems as realistic channels for information transfer it is therefore essential to study the influence of imperfections. Indeed, we have explored the robustness of some PST channels against static perturbations 11, finding that the quality of transfer is often strongly impaired by perturbations. Therefore a question emerges: Is it really necessary to optimize every single interaction in a chain? Can we find simpler systems showing good transfer under perturbations?

In this work we focus on the behavior of essentially homogeneous chains where only the first and last couplings can be adjusted. We show that under perturbations these chains can achieve an optimized state transfer (OST) comparable to or even better than that of fully engineered PST systems. Two interesting regimes for transmission can be observed when the boundary couplings are varied; for unperturbed chains these regimes have already been studied recently $[9,10,21$ 25]. Favorable values for the speed and fidelity of transmission were observed (i) for an optimized (length-dependent) value of the boundary couplings which renders quantum state transfer approximately dispersionless and (ii) in the limit of weak boundary couplings. For both regimes we study the robustness against perturbations, demonstrating that transfer efficiency comparable or better than that of the most robust PST systems can be reached without the demanding engineering of a large number of couplings.

\section{SPIN CHAINS AS STATE TRANSFER CHANNELS}

We consider a spin- $\frac{1}{2}$ chain with XX interactions between nearest neighbors, described by the Hamiltonian

$$
H=\frac{1}{2} \sum_{i=1}^{N-1} J_{i}\left(\sigma_{i}^{x} \sigma_{i+1}^{x}+\sigma_{i}^{y} \sigma_{i+1}^{y}\right),
$$

where $\sigma_{i}^{x, y}$ are the Pauli matrices, $N$ is the chain length, and $J_{i}>0$ is the exchange interaction coupling. We assume the mirror symmetry $J_{i}=J_{N-i}$, which is essential for PST 2, 3]. These spin chains may be modeled with flux qubits 26 28], quantum dots 15, 29, 30], atoms in optical lattices 31 34], and nitrogen vacancy centers in diamond [9].

The goal is to transmit a quantum state $\left|\psi_{0}\right\rangle$ initially stored on the first $\operatorname{spin}(i=1)$ to the last spin of the chain 
$(i=N) .\left|\psi_{0}\right\rangle$ is an arbitrary normalized superposition of the spin down $(|0\rangle)$ and spin up $(|1\rangle)$ states of the first spin, with the remaining spins of the chain initialized in a spin down state. Note that more general initial states can be treated without much additional effort, since the Hamiltonian (10) is equivalent to one of non-interacting fermions. The Hamiltonian (10) conserves the number of up spins, $\left[H, \Sigma_{i} \sigma_{i}^{z}\right]=0$. Therefore the component $|\mathbf{0}\rangle=|00 \ldots 0\rangle$ of the initial state is an eigenstate of $H$ and only the component $|\mathbf{1}\rangle=\left|1_{1} 0 \ldots \ldots .0\right\rangle$ evolves within the one excitation subspace spanned by the basis states $|\mathbf{i}\rangle=\left|0 \ldots 01_{i} 0 \ldots 0\right\rangle$. To evaluate how well an unknown initial state is transmitted, we use the transmission fidelity, averaged over all possible $\left|\psi_{0}\right\rangle$ from the Bloch sphere (see for details, Ref. [5])

$$
F=\frac{f_{N}}{3} \cos \gamma+\frac{f_{N}^{2}}{6}+\frac{1}{2}
$$

where $f_{N}^{2}=\left|\left\langle\mathbf{N}\left|e^{-\frac{i H t}{\hbar}}\right| \mathbf{1}\right\rangle\right|^{2}$ is the fidelity of transfer between states $|\mathbf{1}\rangle$ and $|\mathbf{N}\rangle$ and $\gamma=\arg \left|f_{N}(t)\right|$. Because the phase $\gamma$ can be controlled by an external field once the state is transferred, we consider $\cos \gamma=1$. By the symmetries of the system, this fidelity can be expressed in terms of the single-excitation energies $E_{k}$ and the eigenvectors $\left|\Psi_{k}\right\rangle$ of $H$, in the following way

$$
f_{N}=\left|\sum_{k, s}(-1)^{k+s} P_{k, 1} P_{s, 1} e^{-i\left(E_{k}-E_{s}\right) t}\right|
$$

where $P_{k, 1}=a_{k, 1}^{2}$ are the eigenvector probabilities on the first site of the chain, since $|\mathbf{i}\rangle=\sum a_{k, i}\left|\Psi_{k}\right\rangle$. PST channels are distinguished by commensurate energies $E_{k}$, that is, all transition frequencies share a common divisor to make $f_{N}=1$ in Eq. (3) at a suitable PST time $\tau_{P S T}$ 13, 14]. This condition is obtained by suitably modulating the spin-spin couplings $J_{i}[11,14,16$.

A long unmodulated homogeneous spin channel, $J_{i}=$ $J \forall i$, cannot transfer a state perfectly, since due to the dispersive quantum dynamics the transfer fidelity decreases with the number of spins in the channel [5]. In fact, rigorous PST in a homogeneous chain is possible only for $N \leq 3[13$, 35]. However, transfer can be noticeably improved just by lowering the couplings of the spins at the ends of the channel.

We consider the two surface spins $i=1$ and $N$ interacting with the inner spins with $J_{1}=J_{N-1}=\alpha J$ while the remaining spins compose a homogeneous chain with $J_{i}=J$. We call this Hamiltonian $H^{\alpha}$, where $\alpha \in(0,1]$ is a control parameter. This system has already been studied in Refs. [9, 21, 23 25].

Two regimes for $\alpha$ can be used for OST: (i) the optimal-coupling regime $\left(\alpha=\alpha_{\text {opt }} \sim N^{-\frac{1}{6}}\right)$ possessing an almost equidistant spectrum $E_{k}$ in the middle of the energy band, resulting in a quasi dispersionless fast transfer with high fidelity [10, 24, 25]; and (ii) the weak-coupling regime $(\alpha \ll 1)$. In that regime the transmitted state

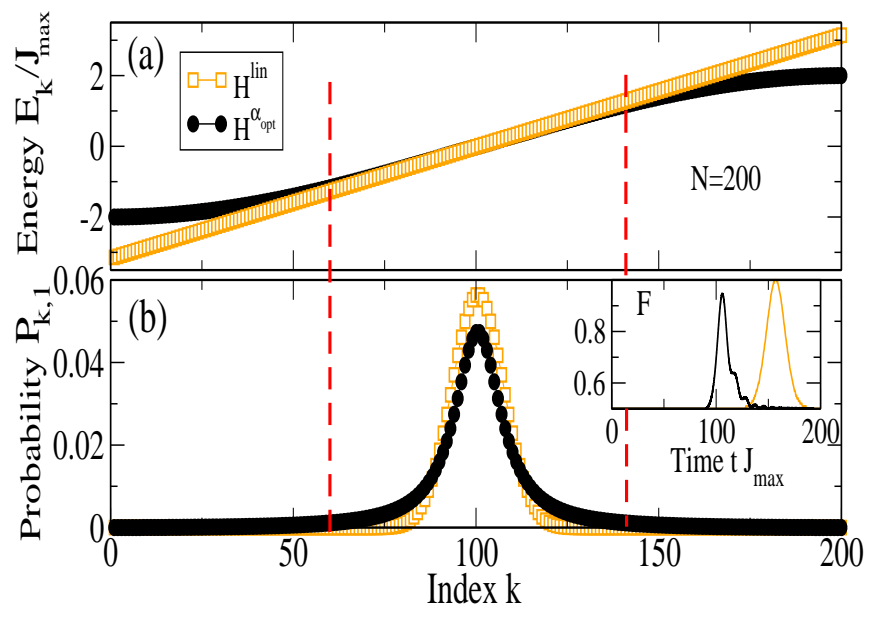

Figure 1: (Color online) Properties of the $H^{\alpha_{o p t}}$ system (black solid dots) and the $H^{\text {lin }}$ system (orange open squares) for a chain length $N=200$. (a) Eigenenergies $E_{k}$. (b) Probabilities $P_{k, 1}$ of the initial state $\left|\psi_{0}\right\rangle=|\mathbf{1}\rangle$. The dashed vertical lines show the dominant energy eigenstates $|k\rangle$ that contribute to the state transfer. $P_{k, 1}^{\alpha_{\text {opt }}}$ is Lorentzian and $P_{k, 1}^{\text {lin }}$ Gaussian. Inset: Evolution of the averaged fidelity of the state transfer.

appears and then reappears roughly periodically at the receiving end of the chain. Almost perfect transfer is achieved with the first arrival due to the fact that only very few eigenstates from the center of the energy band are involved, which are highly localized at the boundaries of the chain [9, 21, 24].

The characteristic features of the two regimes just mentioned were also observed to be essential for the robustness of PST spin-chain channels against perturbations [11. The most robust systems either showed an equidistant (linear) energy spectrum generating the analog of dispersionless wave packet transfer or a large density of states in the center of the band with the corresponding eigenstates localized at the boundary sites of the chain and thus dominating the end-to-end transfer [11]. A class of PST systems is characterized by a power-law spectrum $E_{k}=\operatorname{sgn}(k)|k|^{m}$, where $k=-\frac{N-1}{2}, \ldots, \frac{N-1}{2}$ and the exponent $m$ is a positive integer. We specifically address the linear case, $m=1$, and call the corresponding Hamiltonian $H^{\text {lin }}$ [13], and similarly for the quadratic case, $m=2$, with Hamiltonian $H^{\text {quad }}$ 11].

The OST system described by $H^{\alpha}$, requiring control of only two boundary couplings, would obviously be simpler to implement than the PST systems requiring engineering of all couplings along the chain. In the following we compare the transmission performance of OST and PST systems under the influence of disordered couplings in the channel assuming perfect control of the boundary couplings. We make the same assumption for the engineered chains.

Static disorder in the couplings within the transfer channel is described by $J_{i} \rightarrow J_{i}+\Delta J_{i}(i=2, \ldots, N-2)$, with $\Delta J_{i}$ being a random variable. We consider two possible coupling disorder models: (a) relative static disor- 
$\operatorname{der}(R S D)$ [11, 19, 36], where each coupling is allowed to fluctuate by a certain fraction of its ideal size, $\Delta J_{i}=J_{i} \delta_{i}$, and (b) absolute static disorder (ASD), where all couplings may fluctuate within a certain fixed range which we measure in terms of $J_{\max }=\max J_{i}: \Delta J_{i}=J_{\max } \delta_{i}$ [20]. Each $\delta_{i}$ is an independent and uniformly distributed random variable in the interval $\left[-\varepsilon_{J}, \varepsilon_{J}\right] . \varepsilon_{J}>0$ characterizes the strength of the disorder. The two coupling disorder models are equivalent for the OST systems since all couplings are equal there. However, in the fully engineered PST systems $J_{\max }-J_{\min }$ depends on the type of system and tends to increase with $N$ so that absolute disorder is expected to be more damaging than the relative one in these systems. The relevant kind of disorder depends on the particular experimental method used to engineer the spin chains.

\section{A. Optimal coupling regime}

When $\alpha=\alpha_{o p t}$ in $H^{\alpha}$ the spectrum is linear in the middle of the energy band [Fig. 1(a)]. The probability $P_{k, 1}$ of the $k$ th energy eigenstate to participate in the state transfer is shown in Fig. 1(b) as a function of $k$ for $N=200$. The linear part of the spectrum evidently dominates the dynamics. Also shown in the figure are the corresponding quantities for the linear PST chain. The obvious similarities between these two systems suggest a comparison of their properties in the perturbed case, which is discussed below. The inset in Fig. T(b) shows the averaged transfer fidelity of the unperturbed linear PST and $\alpha_{\text {opt }}$ systems, as a function of time. The maximum fidelity of the $\alpha_{\text {opt }}$ system is clearly smaller than unity, and it decreases with each revival of the signal. However, the transfer time $\tau$ of the $\alpha_{\text {opt }}$ system is shorter: $\tau^{l i n}=\frac{\pi N}{4 J_{\max }}[11,13]$ and $\tau^{\alpha_{o p t}} \sim \frac{N}{2 J_{\max }}$ [24]; hence $\tau^{l i n} \sim \frac{\pi}{2} \tau^{\alpha_{o p t}}$.

The main results of the comparison between the linear PST and $\alpha_{\text {opt }}$ systems are shown in Fig. 2 Figure 2(a) shows the fidelity at time $\tau$ given by the transfer time of the unperturbed case. The transfer fidelity is averaged over the Bloch sphere, as well as over the disorder, for $N=200$, as a function of the disorder strength $\varepsilon_{J}$. As expected the linear PST chain with RSD always performs better than that with ASD. For vanishing disorder strength the linear PST chain yields unit fidelity, which the boundary-controlled chain does not, since its energy spectrum is only approximately, but not strictly linear. The linear PST system with RSD has fidelity higher than that of the boundary controlled system for all $\varepsilon_{J}$, but for $\varepsilon_{J} \gtrsim 0.1$ (where the fidelity is already rather low) the difference in fidelity between the two systems becomes insignificant. However, with ASD , there is a finite perturbation strength $\left(\varepsilon_{J} \approx 0.05\right)$ where the $\alpha_{\text {opt }}$ system becomes better than the linear PST system. Hence, if fidelity very close to unity is desired, complete engineering of the couplings and very good disorder protection are mandatory. However, if only moderate fi- delity is needed (or possible, due to high disorder level) a boundary-controlled system might do.

In order to see how the transfer properties depend on the chain length we show in Figs. 2(b)-2(d) the average fidelity for each of the three systems as a contour and color plot in the $\left(\varepsilon_{J}, N\right)$ plane. The contour lines are straight lines (representing power laws) in most cases, with deviations for the boundary-controlled system at weak disorder. The open circles in Figs. 2(c) and d indicate where the fidelity of the boundary-controlled chain is equal to that of the linear PST chain with ASD; to the right of them the boundary-controlled chain has higher fidelity.

The results above already indicate that there is no simple general answer to the question whether fully engineered or boundary-controlled spin chains provide better quantum state transfer properties in the presence of disorder. The static disorder model, strength and chain length all are important factors in answering that question. We arrive at similar conclusions in our next example.

\section{B. Weak Coupling Regime}

When the boundary spins are only weakly coupled to the channel, i.e., $\alpha J_{\max }=\alpha_{0} J_{\max } \ll \frac{1}{\sqrt{N}}$ in $H^{\alpha}$, an almost perfect state transfer, $F \approx 1-\mathcal{O}\left(\alpha^{2} J_{\max }^{2} N\right)$, is achieved (for details, see Ref. [21]). In this region, the parity of $N$ is relevant. This can be understood by studying the spectral properties of the "channel" of $N-2$ spins connecting the transmitting and receiving qubits. For odd (even) $N$ the dynamics of the channel is dominated by two (three) states situated symmetrically about the center of the energy spectrum [21]. The energy differences between these dominant levels determine the transfer time which is obtained as $\tau_{\text {even }}^{\alpha_{0}} \sim \frac{\pi}{2 \alpha^{2} J_{\max }}$ and $\tau_{\text {odd }}^{\alpha_{0}} \sim \frac{\pi \sqrt{N}}{2 \alpha J_{\max }}$ [21]. Since $\tau$ is $N$ independent for even $N$ and $\alpha_{0} J_{\max }<\frac{1}{\sqrt{N}}$, the transfer is faster for odd $N$. Very similar properties of the energy eigenstates which dominate the state transfer are found in the fully engineered (PST) chain with odd $N$ and a quadratic energy spectrum, which make it the most robust PST system for relative disorder [11]. We therefore compare this system to the boundary-controlled chain at weak coupling. We find that the transfer time of the quadratic PST chain is $\tau^{\text {quad }} \sim \frac{\pi N^{2}}{8 J_{\max }}$ which is longer than $\tau_{\text {odd }}^{\alpha_{0}}$ for $\alpha \gtrsim \frac{4}{N^{3 / 2}}$ for reasonably large $N$.

Figures 3(a) and (b) show the averaged fidelities for $N=200$ and $N=201$, respectively, for the quadratic PST system and the weak-coupling boundary-controlled system, at time $\tau$ determined by the unperturbed cases and for $\alpha=0.01$. Again, as in the linear case, absolute disorder is much more detrimental than relative disorder. This is connected to the fact that the maximum and minimum couplings in the chain may differ by or- 


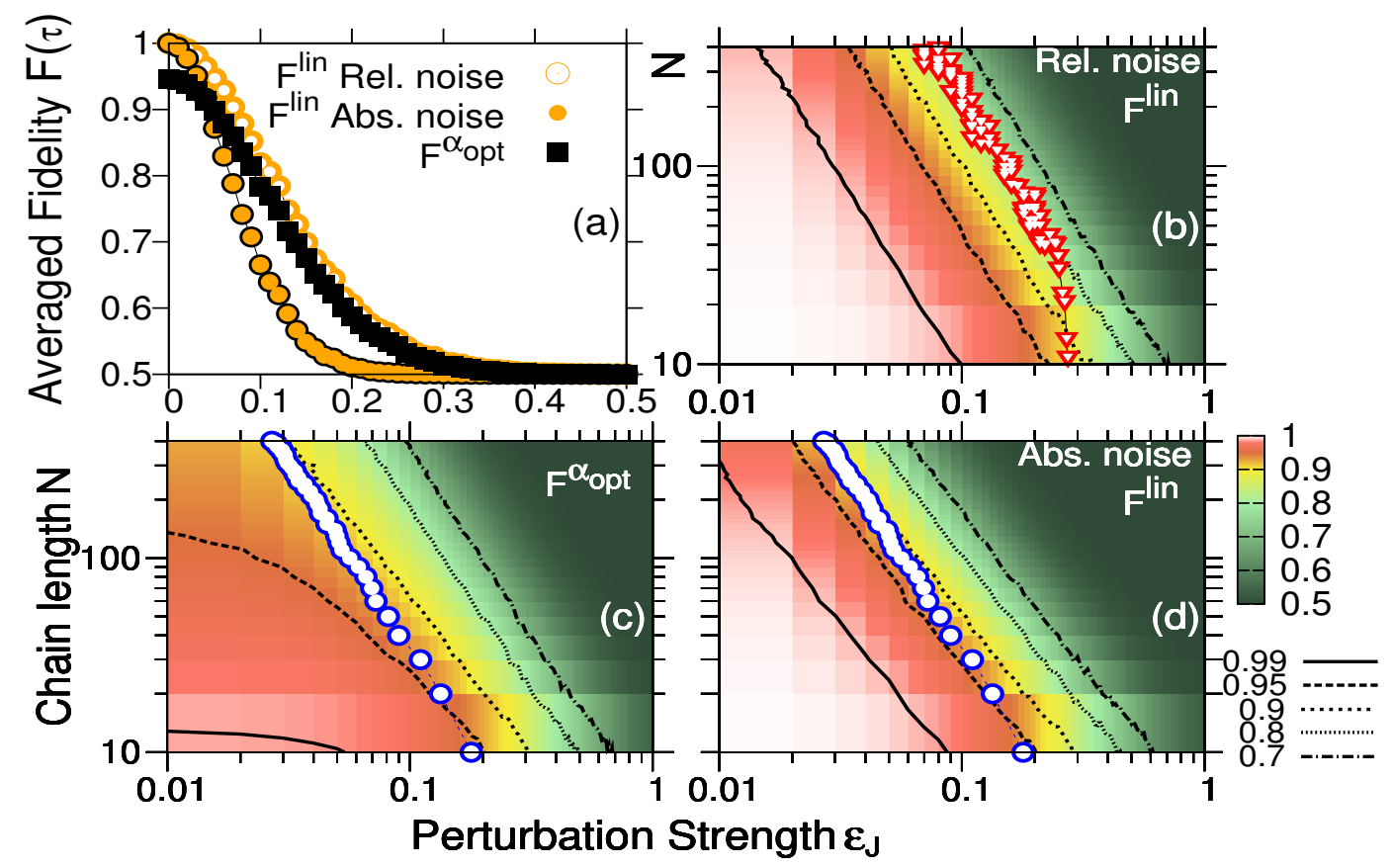

Figure 2: (Color online) Averaged fidelity at time $\tau$ as a function of the perturbation strength $\varepsilon_{J}$ and the chain length $N$ for $H^{\text {lin }}$ and $H^{\alpha_{o p t}}$ systems. Relative and absolute static disorder are considered. (a) $F^{l i n}$ with relative disorder (open circles) and absolute disorder (orange circles) and $F^{\alpha_{o p t}}$ (black squares) for both kinds of disorder when $\mathrm{N}=200$. (b) $F^{\text {lin }}$ with relative disorder. The open triangles indicate when $F^{l i n}=F^{\alpha_{0}}$ shown in Fig 3(c). To the left of the symbols $F^{l i n}>F_{\text {odd }}^{\alpha_{0}}$ (the difference being small, however), while to the right $F^{\alpha_{0}}>F^{l i n}$. (c) $F^{\alpha_{o p t}}$ with both kinds of disorder and (d) $F^{l i n}$ with absolute disorder . The open circles indicate when $F^{\text {lin }}=F^{\alpha_{o p t}}$. To the left of the symbols $F^{\text {lin }}>F^{\alpha_{o p t}}$ and to the right $F^{\alpha_{o p t}}>F^{\text {lin }}$.

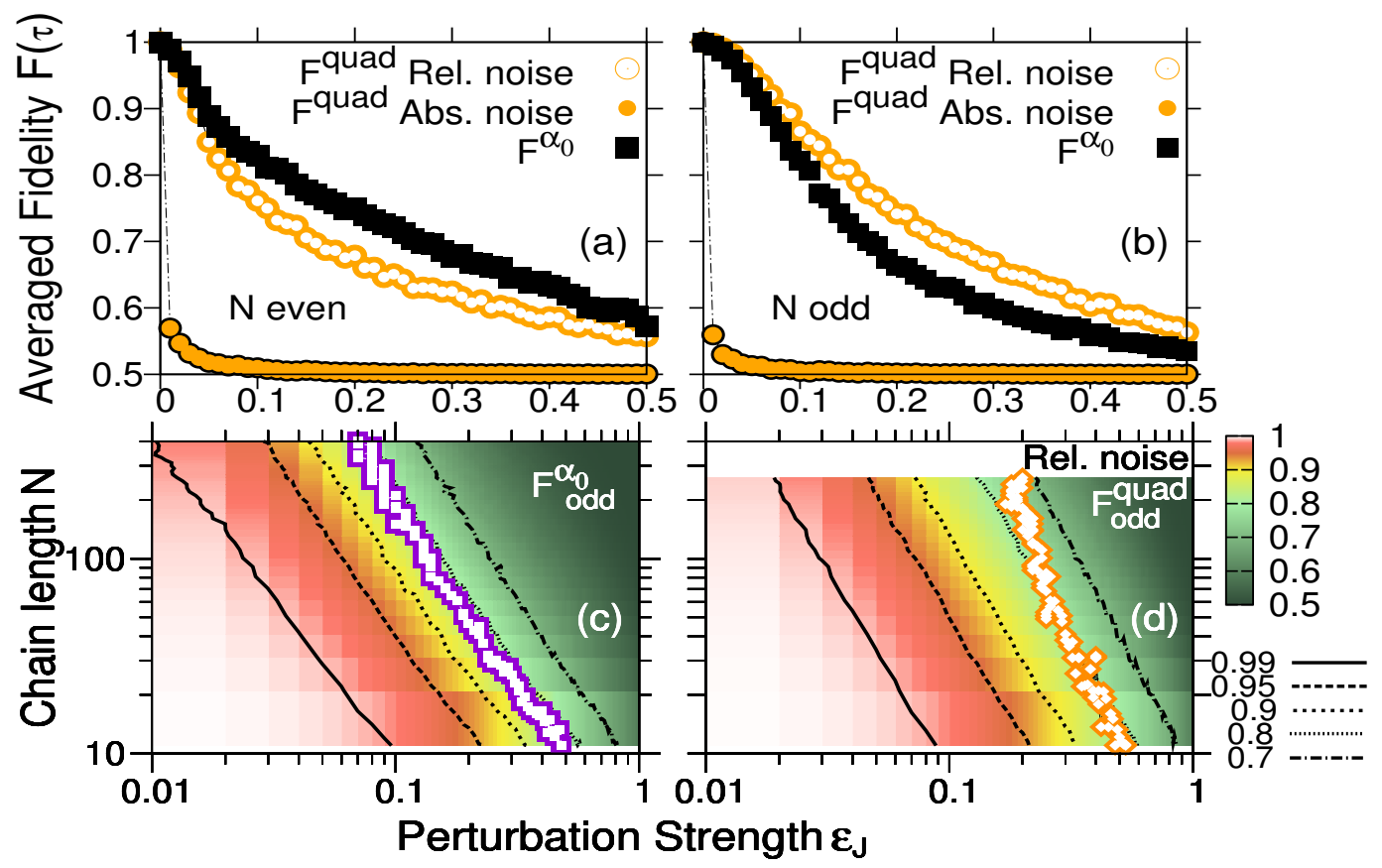

Figure 3: (Color online) Averaged fidelity at time $\tau$ as a function of the perturbation strength $\varepsilon_{J}$ and of the chain length $N$ for $H^{\text {quad }}$ and $H^{\alpha_{0}}$ systems when relative and absolute disorder are considered. (a) $F^{\text {quad }}$ with relative disorder (open circles) and absolute disorder (orange circles) and $F^{\alpha_{0}}$ (black squares) for both kinds of disorder when $N=200$. (b) Same as panel (a) for $N=201$. (c) $F^{\alpha_{0}}$ with both kinds of disorder for odd $N$. The open squares show when $F_{\text {odd }}^{\alpha_{0}}=F_{\text {even }}^{\alpha_{0}}$ (not shown), where $F_{\text {odd }}^{\alpha_{0}}>F_{\text {even }}^{\alpha_{0}}$ to the left of the symbols. (d) $F_{\text {odd }}^{\text {quad }}$ with relative disorder. The open diamonds indicate when $F_{\text {even }}^{\alpha_{0}}=F_{\text {odd }}^{q u a d}$, where $F_{\text {odd }}^{\text {quad }}>F_{\text {even }}^{\alpha_{0}}$ to the left of the symbols. 
ders of magnitude, with the small couplings always close to the ends of the chain [11]. Consequently a fluctuation of a given absolute size may completely spoil the state transport when it affects one of the small couplings close to the boundary. For the boundary-controlled system the two kinds of disorder are again equal by definition. Therefore, for absolute disorder the weak-coupling OST system performs always better than the quadratic PST system. For relative disorder the parity of $N$ matters. The fidelity of the boundary-controlled system is similar or higher (lower) than that of the PST system when $N$ is even (odd). Figures 3(c) and 3(d) show the fidelity as a contour and color plot in the $\left(\varepsilon_{J}, N\right)$ plane for $\alpha=0.01$ and odd $N$. The contour lines are again power laws. The open symbols in Fig. 3 (c) (squares) indicate where the fidelities for odd and even weak-coupling boundarycontrolled systems are equal. To the left of the symbols the fidelity is higher for odd $N$. The open symbols (diamonds) in Fig. 3(d) indicate where the fidelities for odd quadratic PST systems (with relative disorder) and for even weak-coupling boundary-controlled systems are equal. To the left of the symbols the fidelity is higher for the quadratic PST system, but for small perturbation strength differences between the two systems are quite small.

We want to remark that if an actual implementation were to be used, the faulty couplings of the chain could be tested following the recipe given in Ref. 37], which allows the coupling strength estimation of a $X X$ spin chain with an external magnetic field applied to it. In this case the best possible time to remove the state from the chain can be obtained from the numerical integration of the Schrödinger equation, just looking for the smallest time when the fidelity is near 1 . In case the indirect Hamiltonian tomography [38] turns out to be too expensive or cumbersome to perform, the best time to remove the state from the chain is the design time, i.e., the time $\tau$ when the fidelity of the "nonfaulty chain", the one that was intended to be implemented, achieves its best performance.

On the other hand, a detailed analysis of the statistics of the fidelity as a function of time is lacking; so far most studies focus on its average over realizations of the noise. For a particular class of engineered chains [13], De Chiara et al. [19] have shown that the time signal of the fidelity becomes fractal. In this sense, it is difficult to assess how much information is lost because of a bad timing for the readout of the state at the receiving end of the channel.

\section{SUMMARY AND CONCLUSIONS}

For relative disorder, Fig. 4 shows a comparison between all of the systems considered here, linear PST and boundary-controlled with optimal $\alpha_{o p t}$ as well as quadratic PST and weak-coupling $(\alpha=0.01)$ boundarycontrolled, for both even and odd lengths. For each system the figure shows the line in the $\left(\varepsilon_{J}, N\right)$ plane where

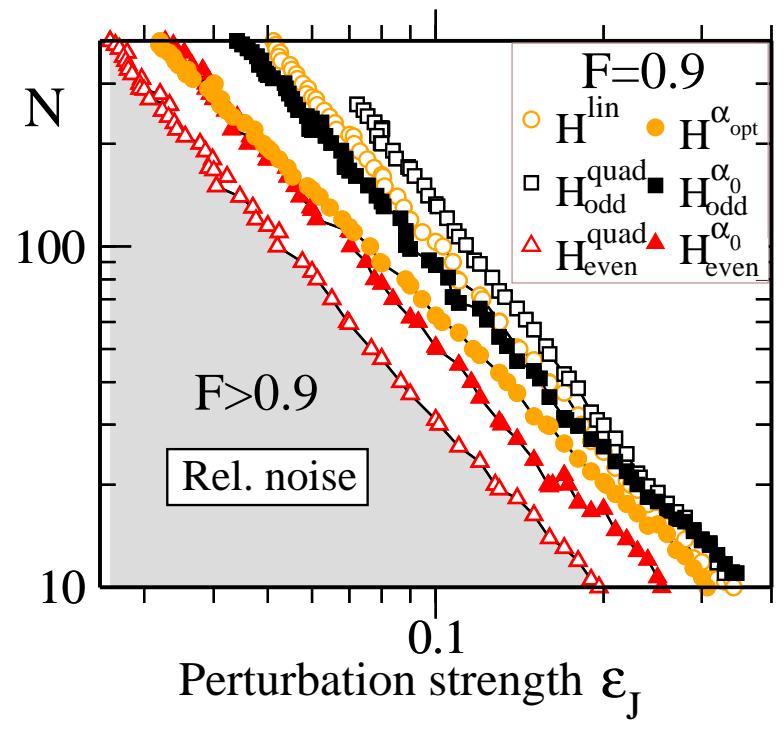

Figure 4: (Color online) Contour lines of the averaged transfer fidelity $F=0.9$ for fully-engineered PST systems (closed symbols) and boundary-controlled $\alpha$-OST systems. To the left of the symbols the transfer fidelity $F>0.9$ for every system.

$F=0.9$. Open symbols denote PST systems; closed symbols correspond to boundary-controlled systems. To the left of the symbols the transfer fidelity of each system is $F>0.9$. It is interesting to note that the lines for the three boundary-controlled systems lie next to each other (at least for long chains), while one of the PST systems (quadratic, even) lies clearly below (performs less well) and the other two PST systems lie slightly above. This situation changes, however, for different levels of fidelity. For example, the $H_{\text {odd }}^{\alpha_{0}}$ system outperforms $H^{l i n}$ in the region to the right of the crossover marked by the open triangles in Fig. 2(b). Note that to the left of that crossover the fidelities of the two systems differ only by up to $4 \%$. On the other hand, to the right of the crossover displayed in Fig. 3(d), $H_{\text {even }}^{\alpha_{0}}$ is the best choice.

For absolute disorder, there is almost always a boundary-controlled system with fidelity larger than that of the PST systems. Only for very small perturbation strength can PST systems be better than OST systems, but the fidelities are similar.

Considering only the PST systems, $H_{\text {odd }}^{\text {quad }}$ performs better than $H^{l i n}$ for relative disorder with similar transfer fidelity for small perturbations. Conversely, $H^{\text {lin }}$ is drastically the more robust choice for absolute disorder. Considering only the OST systems, $H_{o d d}^{\alpha_{0}}$ achieves the highest state transfer fidelities.

For all the channels with $F \rightarrow 1$ in the vanishing perturbation strength limit we find a power law $N \varepsilon_{J}^{\beta}=$ const for the contours of constant fidelity, with $\beta$ near 2, generalizing the fidelity scaling law found for the linear PST system with relative disorder [19]. This quantifies the sensitivity of the channels to perturbations as a function 
of the system size: Increasing the channel length, the transfer fidelity becomes more sensitive to the perturbations.

If the transfer speed is important, independent of the kind of disorder, the faster transfer is achieved by the nonengineered $H^{\alpha_{o p t}}$ system, closely followed by the engineered $H^{l i n}$ system. The other systems are significantly slower.

To summarize, we show that in most situations the transmission performance of boundary-controlled spin chains renders the full engineering of the couplings of a spin chain unnecessary in order to obtain quantum state transmission with high fidelity under static perturbations.

\section{Acknowledgments}

A.Z. and O.O. acknowledge support from SECYTUNC and CONICET and are thankful for the hospitality of Fakultät Physik of the TU Dortmund. A.Z. is thankful for the support of DAAD. We acknowledge useful discussions with Dieter Suter.
[1] D. P. DiVincenzo, Science 270, 255 (1995).

[2] S. Bose, Contemp. Phys. 48, 13 (2007).

[3] A. Kay, Int. J. Quantum Inf. 8, 641 (2010).

[4] R.J. Cook and B.W. Shore, Phys. Rev. A 20, 539 (1979).

[5] S. Bose, Phys. Rev. Lett. 91, 207901 (2003).

[6] T. J. Osborne and N. Linden, Phys. Rev. A 69, 052315 (2004).

[7] D. Burgarth and S. Bose, Phys. Rev. A 71, 052315 (2005).

[8] D. Burgarth, V. Giovannetti and S. Bose, Phys. Rev. A 75, 062327 (2007).

[9] N. Y. Yao, L. Jiang, A. V. Gorshkov, Z.-X. Gong, A. Zhai, L.-M. Duan, and M. D. Lukin, Phys. Rev. Lett. 106, 040505 (2011).

[10] L. Banchi, A. Bayat, P. Verrucchi, S. Bose, Phys. Rev. Lett. 106, 140501 (2011).

[11] A. Zwick, G. A. Álvarez, J. Stolze and O. Osenda, Phys. Rev. A 84, 022311 (2011).

[12] C. Albanese, M. Christandl, N. Datta and A. Ekert, Phys. Rev. Lett. 93, 230502 (2004).

[13] M. Christandl, N. Datta, A. Ekert and A. J. Landahl, Phys. Rev. Lett. 92, 187902 (2004).

[14] P. Karbach and J. Stolze, Phys. Rev. A 72, 030301(R) (2005).

[15] G.M. Nikolopoulos, D. Petrosyan and P. Lambropoulos, J. Phys.: Condens. Matter 16, 4991 (2004)

[16] A. Kay, Phys. Rev. A 73, 032306 (2006).

[17] Y. Wang, F. Shuang, and H. Rabitz, Phys. Rev. A 84, 012307 (2011).

[18] V. Kostak, G. M. Nikolopoulos, and I. Jex, Phys. Rev. A 75, 042319 (2007)

[19] G. De Chiara, D. Rossini, S. Montangero, and R. Fazio, Phys. Rev A 72, 012323 (2005).

[20] R. Ronke, T. P. Spiller and I. D'Amico, Phys. Rev. A 83, 012325 (2011).

[21] A. Wójcik, T. Łuczak, P. Kurzyński, A. Grudka, T. Gdala, and M. Bednarska, Phys. Rev. A 72, 034303
(2005).

[22] L. Banchi, T. J. G. Apollaro, A. Cuccoli, R. Vaia, and P. Verrucchi, Phys. Rev. A 82, 052321 (2010).

[23] E. B. Fel'dman, E. I. Kuznetsova, A. I. Zenchuk, Phys. Rev. A 82, 022332 (2010).

[24] A. Zwick and O. Osenda, J. Phys. A: Math. Theor. 44, 105302 (2011).

[25] L. Banchi, T. J. G. Apollaro, A. Cuccoli, R. Vaia and P. Verrucchi, New J. Phys. 13, 123006 (2011)..

[26] A. Romito, R. Fazio, and C. Bruder, Phys. Rev. B 71, 100501(R) (2005).

[27] F. W. Strauch and C. J. Williams, Phys. Rev. B 78, 094516 (2008).

[28] D. I. Tsomokos, M. J. Hartmann, S. F. Huelga, and M. B. Plenio, New J. Phys. 9, 79 (2007).

[29] G. M. Nikolopoulos, D. Petrosyan, and P. Lambropoulos, Europhys. Lett. 65, 297 (2004).

[30] D. Petrosyan and P. Lambropoulos, Opt. Commun. 264, 419 (2006).

[31] M.J. Hartmann, F.G.S.L. Brandao, and M.B. Plenio, Phys. Rev. Lett. 99, 160501 (2007).

[32] U. Dorner, P. Fedichev, D. Jaksch, M. Lewenstein, and P. Zoller, Phys. Rev. Lett. 91, 073601 (2003).

[33] M. Lewenstein, A. Sanpera, V. Ahufinger, B. Damski, A. Sen, and U. Sen, Adv. Phys. 56, 243 (2007).

[34] L.-M. Duan, E. Demler, and M.D. Lukin, Phys. Rev. Lett. 91, 090402 (2003).

[35] M. Christandl1, N. Datta, T.C. Dorlas, A. Ekert, A. Kay, and A.J. Landahl, Phys. Rev. A 71, 032312 (2005)

[36] D. Petrosyan, G. M. Nikolopoulos, and P. Lambropoulos, Phys. Rev. A 81, 042307 (2010).

[37] M. Wieśniak1, and M. Markiewicz, Phys. Rev. A 81, 032340 (2010).

[38] D. Burgarth, K. Maruyama and F. Nori, New J. Phys. 13, 013019 (2011) 